\title{
Conserved arginine residue in the membrane-spanning domain of HIV-1 gp41 is required for efficient membrane fusion
}

\author{
Yufei Long ${ }^{1,2}$, Fanxia Meng ${ }^{1}$, Naoyuki Kondo ${ }^{1,3}$, Aikichi Iwamoto ${ }^{4}$, Zene Matsuda ${ }^{1,5} \bowtie$ \\ ${ }^{1}$ China-Japan Joint Laboratory of Structural Virology and Immunology, Institute of Biophysics, Chinese Academy of Sciences, 15 \\ Datun Road, Beijing 100101, China \\ ${ }^{2}$ Graduate University of the Chinese Academy of Sciences, Beijing 100101, China \\ ${ }^{3}$ Department of Pediatrics, Emory University School of Medicine, 2015 Uppergate Dr. Atlanta, GA 30322, USA \\ ${ }^{4}$ Division of Infectious Diseases, Advanced Clinical Research Center, University of Tokyo, 4-6-1 Shirokanedai, Minato-ku, Tokyo \\ 108-8639, Japan \\ ${ }^{5}$ Research Center for Asian Infectious Diseases, Institute of Medical Science, University of Tokyo, 4-6-1 Shirokanedai, Minato- \\ ku, Tokyo 108-8639, Japan \\ Correspondence: zmatsuda@ims.u-tokyo.ac.jp
}

Received April 20, 2011 Accepted May 8, 2011

\section{ABSTRACT}

Despite the high mutation rate of HIV-1, the amino acid sequences of the membrane-spanning domain (MSD) of HIV-1 gp41 are well conserved. Arginine residues are rarely found in single membrane-spanning domains, yet an arginine residue, $R^{696}$ (the numbering is based on that of HXB2), is highly conserved in HIV-1 gp41. To examine the role of $R^{696}$, it was mutated to $K, A, I, L, D, E, N$, and $Q$. Most of these substitutions did not affect the expression, processing or surface distribution of the envelope protein (Env). However, a syncytia formation assay showed that the substitution of $R^{696}$ with amino acid residues other than $\mathrm{K}$, a naturally observed mutation in the gp41 MSD, decreased fusion activity. Substitution with hydrophobic amino acid residues (A, I, and L) resulted in a modest decrease, while substitution with $D$ or E, potentially negatively-charged residues, almost abolished the syncytia formation. All the fusion-defective mutants showed slower kinetics with the cell-based dual split protein (DSP) assay that scores the degree of membrane fusion based on pore formation between fusing cells. Interestingly, the D and E substitutions did show some fusion activity in the DSP assays, suggesting that proteins containing $D$ or $E$ substitutions retained some fusion pore-forming capability. However, nascent pores failed to develop, due probably to impaired activity in the pore enlargement process. Our data show the importance of this conserved arginine residue for efficient membrane fusion.

KEYWORDS human immunodeficiency virus, type-1 (HIV-1), gp41, membrane-spanning domain (MSD), arginine, membrane fusion

\section{INTRODUCTION}

Human immunodeficiency virus, type-1 (HIV-1) is a causative agent of the worldwide pandemic of acquired immunodeficiency syndrome (AIDS). The envelope glycoprotein (Env) of HIV-1 plays an essential role in its life cycle. HIV-1 Env is composed of two subunits, gp120 and gp41, which are generated by proteolytic processing of their precursor, gp160, by cellular furin or furin-like proteases (Dubay et al., 1995; Gu et al., 1995). The gp120 subunit recognizes the CD4/ chemokine receptors, and the gp41 subunit induces membrane fusion (Colman and Lawrence, 2003; Weiss, 2003).

The gp41 subunit, a class 1 fusion protein, is divided into three subdomains: an ectodomain, a cytoplasmic domain, and a membrane-spanning domain (MSD). The ectodomain contains an $\mathrm{N}$-terminal fusion peptide followed by coiled-coilforming heptad repeats in the C-terminal region (Melikyan, 2008). The cytoplasmic domain contains amphipathic $\alpha$ helices that modulate membrane fusion activity (Freed and Martin, 1996; Wyss et al., 2005). The MSD consists of a single 
membrane-spanning a-helix composed of about twenty amino acid residues (Haffar et al., 1988; Rowell et al., 1995; Yang et al., 1995; Gangupomu and Abrams, 2010; Liu et al., 2010). The MSD also modulates fusion activity (Helseth et al., 1990; Miyauchi et al., 2005; Welman et al., 2007; Shang et al., 2008; Kondo et al., 2010).

Despite a high mutation rate of HIV-1, the gp41 MSD sequence is highly conserved among different clades (Fig. 1A). As in other transmembrane domains, most amino acid residues in the gp41 MSD are hydrophobic, because charged residues are energetically unfavorable in lipid bilayers (Hessa et al., 2005). However, the gp41 MSD contains a highly conserved and potentially positivelycharged arginine residue near its central region at position $696\left(R^{696}\right)$. Furthermore, the only naturally observed mutation of this arginine residue in HIV-1 isolates is an $R$ to $K$ substitution, suggesting that having a positively-charged residue at this position may be biologically important. This hypothesis is supported by findings in other enveloped viruses, such as foamy virus and hepatitis $C$ virus, where
A. Consensus sequence of MSD of HIV-1 gp41 of different clades.

CONSENSUS_M group
CONSENSUS_A1
CONSENSUS_A2
CONSENSUS_B
CONSENSUS_C
CONSENSUS_D
CONSENSUS_F1
CONSENSUS_F2
CONSENSUS_G
CONSENSUS_H

\author{
yikIFIMIVGGLIGLRIVFAVLSIVnrV \\ yikIFIMIVGGLIGLRIVFAVLSVInrV \\ yirIFIMIVGGLIGLRIVIAIISVVnrV \\ yikIFIMIVGGLVGLRIVFAVLSIVnrV \\ YikIFIMIVGGLIGLRIIFAVLSIVnYV \\ yikIFIMIVGGLIGLRIVEAVLSLVnrV \\ yikIFIMIVGGLIGLRIVFAVLSIVnrV \\ yikIFIMIVGGLIGLRIVFAVLSVVnrV \\ yikIFIMIVGGLIGLRIVFAVLSIVnrV
}
yikIFIMIVGGLIGLRIIFAVLSIVnrV

\section{B. Mutants list}

\begin{tabular}{|c|c|c|}
\hline Wildtype (WT) & WT & yikLFIMIVGGLVGLRIVEAVLSIVnrV \\
\hline Positively charged amino acid & RK & yikLFIMIVGGLVGL트FAVLSIVnrv \\
\hline \multirow[t]{2}{*}{ Negatively charged amino acid } & $\mathrm{RD}$ & yikLFIMIVGGLVGLDIVFAVLSIVnrv \\
\hline & $\mathrm{RE}$ & yikLFIMIVGGLVGLE्EIVFAVLSIVnrV \\
\hline \multirow[t]{5}{*}{ Uncharged amino acid } & $\mathrm{RA}$ & yikLFIMIVGGLVGLA_IVFAVLSIVnrv \\
\hline & $\mathrm{RI}$ & yikLFIMIVGGLVGLIIVFAVLSIVnrv \\
\hline & RL & yikLFIMIVGGLVGLLIVFAVLSIVnrv \\
\hline & $\mathrm{RN}$ & yikLFIMIVGGLVGLNIVFAVLSIVnrv \\
\hline & $\mathrm{RQ}$ & yikLFIMIVGGLVGL金VEAVLSIVnrV \\
\hline
\end{tabular}

Figure 1. Conservation of the arginine residue in the MSD region among different clades of HIV-1 and Arg-substitution mutants used in this study. (A) Consensus sequences of HIV-1 gp41 MSD of different clades were from the Los Alamos HIV sequence database (http://www.hiv.lanl.gov/content/index). The predicted MSD is indicated with capital letters. (B) Substitution mutations used in this study. Mutants were constructed using a QuikChange site mutation kit based on the HXB2 strain. Substitute amino acid residues are underlined. Lysine is potentially positively-charged, like arginine. Aspartic acid and glutamic acid have a negatively-charged side chain. Asparagine and glutamine share the same backbone with aspartic acid and glutamic acid, respectively, but lack their negative charge. Alanine, leucine and isoleucine were chosen to represent uncharged amino acids. 
potentially charged residues in their transmembrane proteins perform critical functions in the biogenesis or fusion activity of Env (Pietschmann et al., 2000; Ciczora et al., 2005). Recently, we have shown that the relationship between $\mathrm{R}^{696}$ and the preceding GXXXG motif in gp41 MSD is important for the biosynthesis and function of HIV-1 Env (Miyauchi et al., 2010).

Studies concerning the conserved arginine residue, however, showed some inconsistencies in the observed phenotypes of the substitution mutants. Replacement of the arginine residue with isoleucine did not affect the infectivity of the mutant virus (Wilk et al., 1996), while replacement with leucine, which is chemically similar to isoleucine, resulted in a drastic loss of fusion activity (Owens et al., 1994). The possibility that these discrepancies arose from different assay systems and different strains of HIV-1 used in the studies cannot be ruled out.

To reveal the importance of the conserved arginine residue to the function of HIV-1 Env, we have generated several substitution mutants of the arginine residue and compared their phenotypes under identical assay conditions with the same HIV-1 strain, HXB2. Specifically, we generated RK, RA, $\mathrm{RI}, \mathrm{RL}, \mathrm{RD}, \mathrm{RN}, \mathrm{RE}$, and $\mathrm{RQ}$ mutations and analyzed their effects on the processing, surface expression, and membrane fusion activity of Env. All the mutations caused little effect on the expression and surface distribution of HIV-1 Env. Positively-charged residues were preferable for efficient membrane fusion compared with substitution with hydrophobic residues, whereas negatively-charged residues severely restricted fusion activity in the cell-cell fusion assay.

\section{RESULTS}

\section{Envelope expression and processing of R-substitution mutants are similar to those of wild type}

To examine the effect of $R^{696}$ substitution on the function of Env, we used several amino acids with different chemical properties to construct a series of substitution mutants (Fig. 1B). The mutant Env was expressed with the mammalian expression vector, pElucEnv, which co-expresses HIV-1 HXB2 Env and an EGFP-firefly luciferase fusion protein as described before (Miyauchi et al., 2005).

Our previous work showed that some mutations introduced into the MSD affected the processing of Env (Miyauchi et al., 2010). Thus, we first examined the protein profile of the present mutants by transfecting COS-7 cells with the expression vectors. A similar level of expression and processing of Env was observed for all the R-substitution mutants except for the RE mutant, which showed a slightly decreased level of processing (Fig. 2). Although $R^{696}$ is highly conserved, substitutions with amino acid residues with different chemical properties seem to have little effect on envelope biosynthesis and processing.

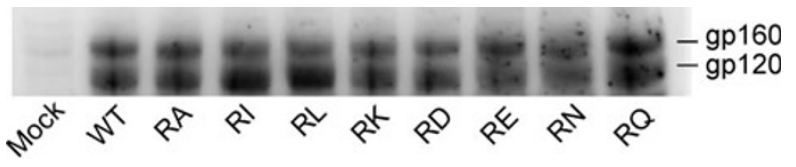

Figure 2. Protein profiles of the R-substitution mutants. The envelope proteins expressed in COS-7 cells transiently transfected with the Env expression vectors for WT or mutants were detected with anti-gp120 polyclonal antibody. The gp160 and gp120 bands are indicated.

\section{Substitution of $R^{696}$ with amino acids other than lysine impairs fusion activity in the syncytia formation assay}

Because many mutations in the MSD affect fusion processes (Helseth et al., 1990; Owens et al., 1994; Miyauchi et al., 2005; Welman et al., 2007; Shang et al., 2008), we determined the effect of the substitutions on the efficiency of membrane fusion using a syncytia formation assay. Env expression vectors carrying different substitution mutants were transfected into 293CD4 cells. Fusion efficiency was expressed with an arbitrary fusion index which incorporates both the number and size of syncytia as described previously (Miyauchi et al., 2005). The fusion indexes of the wild type (WT) and the RK mutant at $24 \mathrm{~h}$ post-transfection were similar, while index scores for $R A, R I, R L, R N$, and $R Q$ mutants showed that their fusion activities decreased by $30 \%-70 \%$ compared with WT (Fig. 3A). Almost no syncytium was observed for RD or RE mutants. Although inefficient processing of the RE mutant may partially account for this defect in fusion activity, results of the RD mutant suggest that defective processing of Env does not fully explain the impact on fusion.

We failed to observe a drastic reduction in the fusion activity of RL mutants as reported previously by Owens et al. (Owens et al., 1994). They used mutants containing an additional mutation (S713T); however, introduction of this additional mutation did not alter fusion activity in our syncytia formation assay (Fig. S1). This difference may derive from the use of different assay systems, in particular different target cell lines. Our 293CD4 cells are selected for their high expression levels of CD4. This may compensate for the attenuated fusion activity, because the levels of CD4 and CXCR4 are known to affect fusion efficiency (Kabat et al., 1994; Kitchen and Zack, 1997; Reeves et al., 2002). Indeed, 293CD4 cells form much larger syncytia than HeLaCD4 cells.

We also observed that the RI and RL mutants possessed a similar fusion activity (Fig. 3A), which might be expected given the similarity in their chemical structures. Although it has been reported that the RI mutant does not affect virus infectivity in an infection assay, the RI mutant clearly showed decreased fusion efficiency compared with WT in our fusion assay. However, the relatively mild reduction in fusion activity may not be translated into an appreciable difference in the infection assay. 

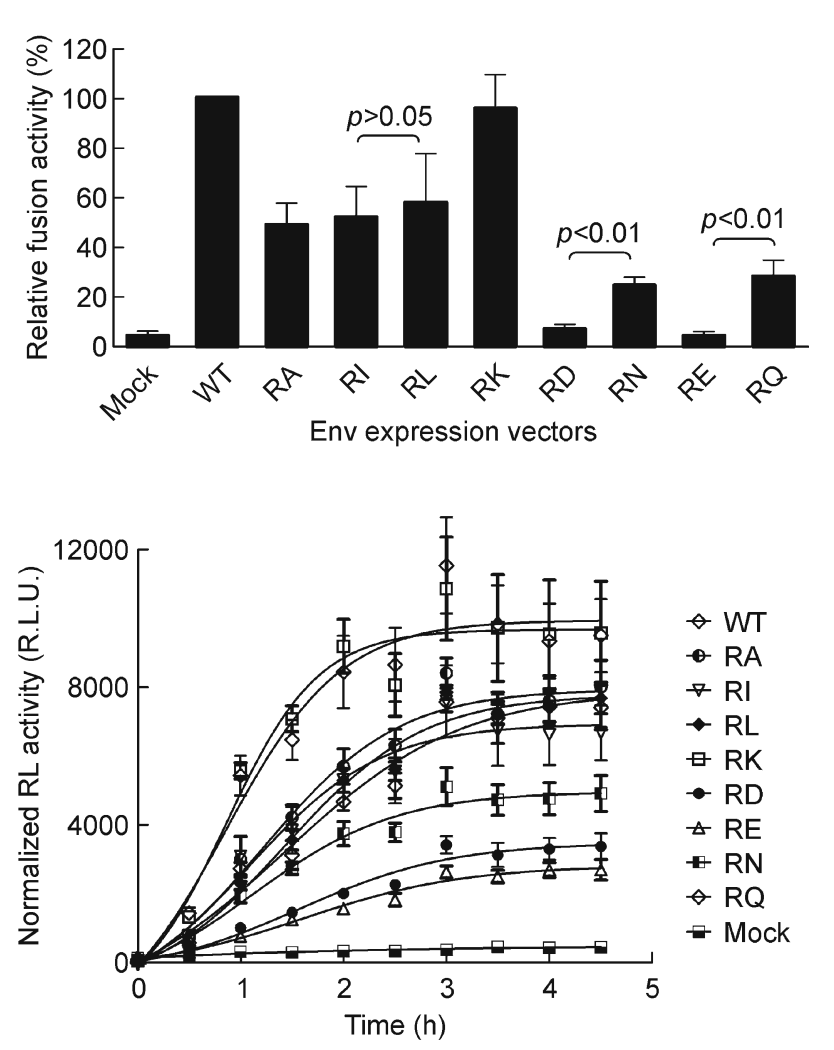

Figure 3. Fusion activity and fusion kinetics of Rsubstitution mutants in the cell-cell fusion assay. (A) The fusion activity was measured by syncytia formation assay. 293CD4 cells were transfected with different mutants or WT, as well as Env KO as a negative control. Nuclei of cells were stained with Hoechst. Relative fusion activity was quantified by using a fusion index (fusion index $=2 x+y$, where $x$ is the number of multinucleated cells [number of nuclei $\geqslant 5$ in five visual fields] and $y$ is the number of multinucleated cells [number of nuclei $<5$ in five visual fields]). Fusion activities for each mutant are shown after normalization to that of WT (with WT activity set normalized to $100 \%$ ). Similar results were obtained from three independent experiments. Statistical significance of the difference between the RI and RL, RD and $R N, R E$ and $R Q$ mutants, respectively was determined using $t$-test. (B) DSP assay was performed to monitor the kinetics of pore formation. Renilla luciferase $(R L)$ activity was measured from $0-5 \mathrm{~h}$ after co-culture. Firefly luciferase (FL) activity from parallel 293FT cells was measured to normalize transfection efficiency. RL activity normalized by $\mathrm{FL}$ activity was shown to reflect the fusion kinetics.

\section{All R-substitution mutants except RK exhibit slower fusion kinetics}

We observed syncytia formation at several time points after transfection. Small syncytia started to appear in cells transfected with either WT or the RK mutant at $20 \mathrm{~h}$ posttransfection. However, the emergence of syncytia in cells transfected with the RA, RI, RL, RN, or RQ mutants did not occur until at least $21-22 \mathrm{~h}$. The time required to reach the maximum syncytia formation for the cells transfected with $R A$, $\mathrm{RI}, \mathrm{RL}, \mathrm{RN}$, or $\mathrm{RQ}$ mutants was much longer than that required for WT- or RK-transfected cells. Syncytia formation for the RD and RE mutants was very inefficient and very few syncytia were observed even at $40 \mathrm{~h}$ post-transfection. Thus, all the substitution mutants other than RK exhibited delayed fusion kinetics.

To obtain a more detailed kinetic profile of membrane fusion for these mutants, we employed the dual split protein (DSP) assay, which can measure real-time pore formation during cell-cell fusion (Kondo et al., 2010). As shown in Fig. 3B, the kinetic profile for the RK mutant was similar to that of WT. Consistent with the syncytia formation assay, we found that mutants producing less syncytia also showed slower kinetics of membrane fusion. However, in contrast to the results of syncytia assay, the RD mutant did show fusion activity in the DSP assay, albeit at a much lower level than that of WT. Thus, it seems that the RD and RE mutants retain an attenuated ability to induce pore formation, but are unable to progress to subsequent steps (e.g., pore dilatation), which may explain the poor syncytia formation in 293CD4 cells transfected with these mutants.

\section{R-substitution mutants do not affect cell surface expression of Env}

The observed fusion activity (Fig. 3A) of the mutants did not correlate well with the total level of Env expression determined by immunoblotting (Fig. 2). Since transport of Env to the cell surface is essential for membrane fusion in our cell-based fusion assay, we examined the surface expression level of mutant Env. Surface gp120 in the transfected cells was detected by an immunofluorescence assay using an antigp120 monoclonal antibody, 2G12, followed by staining with an allophycocyanin (APC)-conjugated secondary antibody. The EGFP signal from Env-expressing vectors indicated transfected cells, and the fluorescence intensities of APC reflected the surface expression level of gp120. Images were captured with a confocal microscope and INCell Analyzer. Representative results of confocal microscopy from WT and selected mutants (RI and RD) are shown in Fig. 4 (others are shown in Fig. S2). The intensities of the APC signals in individual cells transfected with R-substitution mutants were similar to that of WT. The mean fluorescence intensities of APC signals in cells acquired randomly with the INCell Analyzer also showed similar fluorescence levels between WT and the different mutants (Table S1). Thus, it appears that substitution of $\mathrm{R}^{696}$ does not affect the surface distribution of Env, and that the inefficient membrane fusion observed in some of the mutants was not due to the failure of Env to reach the cell surface, but rather due to an intrinsic inability to complete the membrane fusion process. 


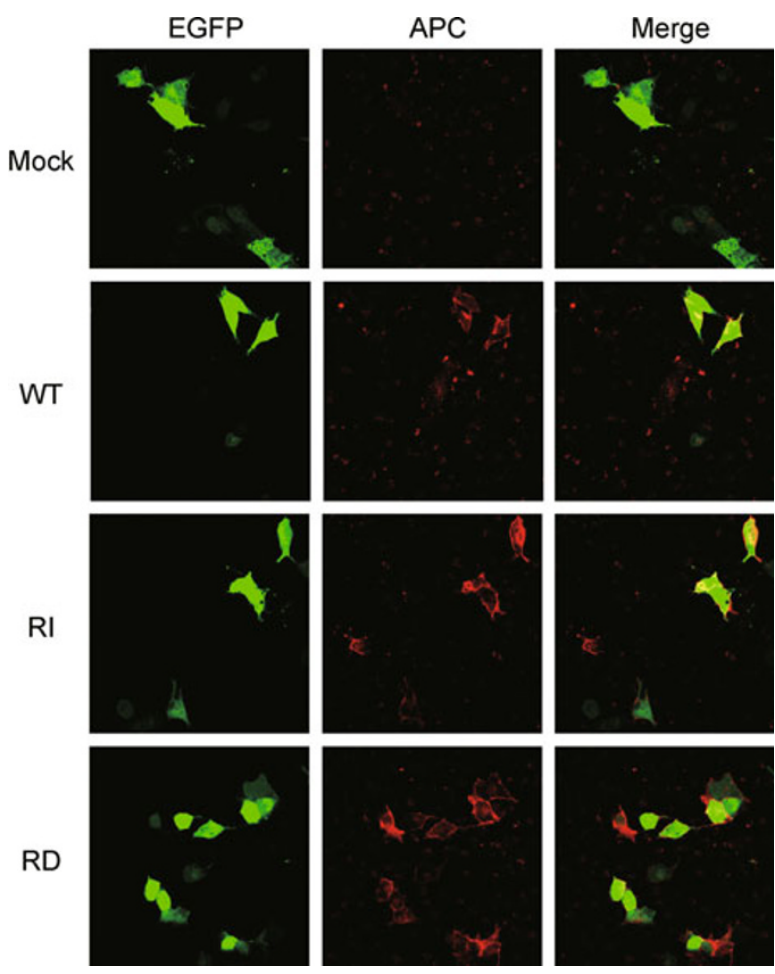

Figure 4. Level of surface expression of R-substitution mutants. COS-7 cells were transfected with WT or mutants and subjected to immunofluorescence assays without permeabilization. Surface-expressed gp120 was detected by staining with $2 \mathrm{G} 12$ primary antibody and APC-conjugated secondary antibody. Internal EGFP signal was used as an indicator of transfected cells. Representative confocal microscopic images are shown.

\section{DISCUSSION}

In this study, we replaced the conserved arginine residue, $\mathrm{R}^{696}$, in the MSD region of HIV-1 gp41 with amino acid residues with different chemical properties, and examined the effect of these substitutions. The substitution of arginine with hydrophobic isoleucine and leucine residues, which are commonly observed in many MSDs, resulted in a modest decrease in fusion activity. Substitution with lysine, a potentially positively-charged residue like arginine, maintained the fusion activity, while substitution with histidine manifested a moderately reduced fusion activity (data not shown). These results suggest that there is some preference for positively-charged residues at position 696 .

When we tested substitutions with potentially negativelycharged residues such as aspartic acid and glutamic acid, we found that the fusion activity of Env in cell-cell fusion assay was almost completely abolished, demonstrating that positive not negative charge seems to be preferred. Moreover, substitutions with asparagine and glutamine, which have similar backbones to aspartic acid and glutamic acid but without negative charges, partially rescued membrane fusion capacity. These results imply that the presence of a potentially positive charge ( $R$ or $K$ ) at position 696 is favorable, the presence of a polar residue $(\mathrm{N}$ or $\mathrm{Q})$ is tolerable, but a negative charge in this position is deleterious to fusion activity.

Although potentially negatively-charged amino acid residues in the MSD are energetically unfavorable (Hessa et al., 2005) and may hinder membrane insertion of the mutant MSD, our analysis of the protein profile by immunoblotting and the immunofluorescence assay ruled out the failure of the mutant Env to incorporate into lipid bilayers as a main cause for this finding. However, our finding does not mean that a negatively-charged residue can be accommodated anywhere in the membrane-spanning domain of HIV-1 gp41 without any destabilizing effect. Indeed the biosynthesis and processing of Env were drastically affected when $D$ was introduced at positions 690,691 and 694 , on the opposite side of the gp41 MSD helix to $\mathrm{R}^{696}$ (Fig. S3).

Because substitution of $\mathrm{R}^{696}$ with negatively-charged residues ( $D$ and $E$ ) had mild negative effects on biosynthesis, processing, and the level of surface expression of Env (Fig. 2 and 4, and Table S1), these mutants have defect(s) in their membrane fusion process. Although the exact mechanism for the observed impairment of fusion activity has yet to be delineated, it is likely that these mutants have defects in postreceptor binding steps, because all of the reported mutations in gp41 MSD had little effect on CD4 binding capacity (Helseth et al., 1990; Miyauchi et al., 2005; Shang and Hunter, 2010). Our DSP assay results (Fig. 3B) suggest that these mutants have a defect in one or more of the steps after initial pore formation.

It is also unclear why the presence of potentially positivelycharged residues ( $R$ and $K$ ) at position 696 facilitates membrane fusion. Although it is uncertain whether these residues are actually charged in the MSD environment ( $\mathrm{Li}$ et al., 2008), a potentially positive charge may interact with water molecules and facilitate their introduction into the hydrophobic core of the MSD. This will destabilize the membranes and may facilitate the process of pore formation (Gangupomu and Abrams, 2010).

Generally, charged residues, be they positively or negatively charged, are rather rare in an MSD because they are energetically unfavorable (Hessa et al., 2005). They can exist in an MSD if they are neutralized with opposite charges by an ionic pairing. Such pairings are well documented in the T-cell receptor- and Fc receptor-complexes (Helseth et al., 1990; Morton et al., 1995). In the case of the gp41 MSD, however, the presence of such a counterpart is undocumented. A recent model predicts that the arginine residues interact with each other within the MSD trimer rather than with residues in foreign MSD (Kim et al., 2009).

Another potential function for a positively-charged amino acid residue in the MSD is to retain the nascent protein in the 
endoplasmic reticulum (ER) (Davis and Hunter, 1987; Bonifacino et al., 1991). Our previous study suggested that alteration of the phase between R and the GXXXG motif in the gp41 MSD a-helix affects its intracellular distribution (Miyauchi et al., 2010). However, all of our R-substitution mutants exhibited a similar level of processing and surface expression. In this regard, it seems that a positively-charged residue in this particular position in gp41 MSD does not have a strong retention function. Nevertheless, a rather prolonged folding of HIV-1 Env due to extensive disulfide bond formation and/or glycosylation may mask any potential retention effect of these positively-charged residues.

In summary, we found here that arginine, a potentially positively-charged residue in the MSD of HIV-1 gp41, was required for efficient membrane fusion. Substitution of the arginine residue with either hydrophobic or uncharged polar residues resulted in decreased fusion activity. Substitution with potentially negatively-charged residues completely abolished fusion activity. The impairment in these mutants seems to be in the post-receptor binding steps, probably in the pore dilatation process.

\section{MATERIALS AND METHODS}

\section{Plasmid construction}

The QuickChange Site-Directed Mutagenesis kit (Stratagene, La Jolla, CA) was used to generate the mutants in this study. The plasmid, pGEM7zNB, which contains the 1.2-kb Nhel-BamHI fragment covering the env portion of HXB2RU3 $\Delta \mathrm{N}$, was used as a template as described previously (Miyauchi et al., 2005). Complementary oligonucleotide pairs containing the substitution codon were used to introduce the site-directed mutation. We used the following codons: GCA for the RA mutation; ATC for the RI mutation; CTG for the RL mutation; AAA for the RK mutation; GAT for the RD mutation; GAA for the RE mutation; AAT for the RN mutation; CAA for the RQ mutation; and ACA for the S713T mutation. The sequenced mutated fragment was cloned into the pElucEnv plasmid as a Nhel-BamHI fragment. The pElucEnv plasmid, which contains EGFP-firefly luciferase gene as a transfection marker, was used to express Env in mammalian cells.

\section{Cell culture and transfection}

COS-7 cells, 293FT cells (Invitrogen, Carlsbad, CA) or 293CD4 cells (293 cells constitutively expressing human CD4) were grown in Dulbecco's modified Eagle's medium (Sigma, St. Louis, MO) supplemented with $10 \%$ fetal bovine serum (HyClone). Cells were kept under $5 \% \mathrm{CO}_{2}$ in a humidified incubator (SANYO, Japan). Cells were seeded into a 6-well or 96-well plate (BD Falcon) one day prior to transfection with FuGENE HD transfection reagent (Roche Diagnostic, Indianapolis, IN).

\section{Immunoblotting}

COS-7 cells $\left(2 \times 10^{5}\right)$ were transiently transfected with $2 \mu \mathrm{g}$ DNA of pElucEnv containing WT or mutant constructs using FuGENE HD in a 6-well culture plate. Forty-eight hours after transfection, the cells were lysed with the RIPA lysis buffer (Thermo Fisher Scientific, Madison, $\mathrm{WI})$ for SDS-PAGE analysis. Meanwhile, aliquots $(50 \mu \mathrm{L})$ from a $1 \mathrm{~mL}$ pre-lysis cell suspension were taken to measure the firefly luciferase (FL) activity. After normalization for FL activity, an appropriate amount of each cell lysate was electrophoresed (10\% SDS-PAGE, Bio-Rad Ready-Gel J) and transferred to a polyvinylidene fluoride membrane (Millipore, Immobilon- $\mathrm{P}^{\mathrm{SQ}}$ ). The blot was probed with anti-gp120 polyclonal antibody (Fitzgerald, Concord, MA). Anti-goat immunoglobulin (Amersham) was used as the secondary antibody. The blot was further treated with a streptavidin-horseradish peroxidase conjugate (GE Healthcare Bio-Sciences $A B$ ) and Lumi-Light ${ }^{P L U S}$ (Roche, Indianapolis, IN). Images were obtained with LAS3000 (Fujifilm, Tokyo, Japan).

\section{Fusion assay (syncytia formation)}

The 293CD4 cells were transfected with pElucEnv mammalian expression vector, and the formation of syncytia was observed by microscopy at 20-24 $\mathrm{h}$ post-transfection. Hoechst staining was used to visualize the nuclei for calculating the fusion index (Miyauchi et al., 2005). The fusion index reflects both the number and size of syncytia observed in five randomly selected fields (fusion index $=2 x+y$, where $x$ is the number of multinucleated cells [number of nuclei $\geqslant 5$ ] and $y$ is the number of multinucleated cells [number of nuclei $<5]$ ).

\section{DSP assay}

The DSP assay was performed as described previously (Kondo et al., $2010,2011)$. Briefly, $293 \mathrm{FT}$ cells $\left(1.3 \times 10^{4}\right.$ per well $)$ and $293 \mathrm{CD} 4$ cells $\left(9 \times 10^{5}\right.$ per dish) were prepared in a 96 -well plate and the UpCell dish (6 $\mathrm{cm}$ in diameter, Nunc), respectively one day prior to transfection. $\mathrm{pDSP}_{1-7}$ and $\mathrm{pElucEnv}$ expression vector were co-transfected into 293FT cells, and pDSP $_{8-11}$ was transfected into 293CD4 cells. Thirtysix hours post-transfection, 293CD4 cells were co-cultured with 293FT, and the Renilla luciferase (RL) activity was measured by GloMax Luminometer (Promega, Madison, WI) for $0-5 \mathrm{~h}$ after coculture. The firefly luciferase (FL) activity measured with similarly prepared 293FT cells was used to normalize the transfection efficiency.

\section{Immunofluorescence assay}

Immunofluorescence assays (IFA) without permeabilization were used to determine the cell surface expression level of the envelope proteins. After fixing with $2 \%$ paraformaldehyde (PFA), cells were immunostained with monoclonal antibody (2G12) and secondary allophycocyanin (APC)-conjugated anti-human IgG antibody (Jackson ImmunoResearch, Amish country, PA). Only surface-expressed Env can be stained. Internal EGFP signal was used as a transfection marker. Images of stained cells were captured with an INCell Analyzer 1000 (GE Healthcare, Fairfield, CT) under $10 \times$ objective lens and a confocal microscope (Olympus, FV1000) under $40 \times$ objective lens.

\section{ACKNOWLEDGEMENTS}

This work was supported by the Ministry of Education, Culture, Sports, Science and Technology of Japan, Japan Initiative for Global 
Research Network on Infectious Diseases (J-GRID). We thank Dr. Kunito Yoshiike for his critical reading of the manuscript.

\section{ABBREVIATIONS}

APC, allophycocyanin; DSP, dual split protein; Env, envelope glycoprotein; FL, firefly luciferase; HIV-1, human immunodeficiency virus, type-1; IFA, immunofluorescence assay; MSD, membranespanning domain; RL, Renilla luciferase; TCR, T cell receptor; WT, wild type

\section{REFERENCES}

Bonifacino, J.S., Cosson, P., Shah, N., and Klausner, R.D. (1991). Role of potentially charged transmembrane residues in targeting proteins for retention and degradation within the endoplasmic reticulum. EMBO J 10, 2783-2793.

Ciczora, Y., Callens, N., Montpellier, C., Bartosch, B., Cosset, F.L., Op de Beeck, A., and Dubuisson, J. (2005). Contribution of the charged residues of hepatitis $C$ virus glycoprotein E2 transmembrane domain to the functions of the E1E2 heterodimer. J Gen Virol 86, 2793-2798.

Colman, P.M., and Lawrence, M.C. (2003). The structural biology of type I viral membrane fusion. Nat Rev Mol Cell Biol 4, 309-319.

Davis, G.L., and Hunter, E. (1987). A charged amino acid substitution within the transmembrane anchor of the Rous sarcoma virus envelope glycoprotein affects surface expression but not intracellular transport. J Cell Biol 105, 1191-1203.

Dubay, J.W., Dubay, S.R., Shin, H.J., and Hunter, E. (1995). Analysis of the cleavage site of the human immunodeficiency virus type 1 glycoprotein: requirement of precursor cleavage for glycoprotein incorporation. J Virol 69, 4675-4682.

Freed, E.O., and Martin, M.A. (1996). Domains of the human immunodeficiency virus type 1 matrix and gp41 cytoplasmic tail required for envelope incorporation into virions. J Virol 70, 341-351.

Gangupomu, V.K., and Abrams, C.F. (2010). All-atom models of the membrane-spanning domain of HIV-1 gp41 from metadynamics. Biophys J 99, 3438-3444.

Gu, M., Rappaport, J., and Leppla, S.H. (1995). Furin is important but not essential for the proteolytic maturation of gp160 of HIV-1. FEBS Lett 365, 95-97.

Haffar, O.K., Dowbenko, D.J., and Berman, P.W. (1988). Topogenic analysis of the human immunodeficiency virus type 1 envelope glycoprotein, gp160, in microsomal membranes. J Cell Biol 107, 1677-1687.

Helseth, E., Olshevsky, U., Gabuzda, D., Ardman, B., Haseltine, W., and Sodroski, J. (1990). Changes in the transmembrane region of the human immunodeficiency virus type 1 gp41 envelope glycoprotein affect membrane fusion. J Virol 64, 6314-6318.

Hessa, T., Kim, H., Bihlmaier, K., Lundin, C., Boekel, J., Andersson, H., Nilsson, I., White, S.H., and von Heijne, G. (2005). Recognition of transmembrane helices by the endoplasmic reticulum translocon. Nature 433, 377-381.

Kabat, D., Kozak, S.L., Wehrly, K., and Chesebro, B. (1994). Differences in CD4 dependence for infectivity of laboratoryadapted and primary patient isolates of human immunodeficiency virus type 1. J Virol 68, 2570-2577.

Kim, J.H., Hartley, T.L., Curran, A.R., and Engelman, D.M. (2009). Molecular dynamics studies of the transmembrane domain of gp41 from HIV-1. Biochim Biophys Acta 1788, 1804-1812.

Kitchen, S.G., and Zack, J.A. (1997). CXCR4 expression during lymphopoiesis: implications for human immunodeficiency virus type 1 infection of the thymus. J Virol 71, 6928-6934.

Kondo, N., Miyauchi, K., and Matsuda, Z. (2011). Monitoring viralmediated membrane fusion using fluorescent reporter methods. Curr Protoc Cell Biol Chapter 26, Unit 26.9.

Kondo, N., Miyauchi, K., Meng, F., Iwamoto, A., and Matsuda, Z. (2010). Conformational changes of the HIV-1 envelope protein during membrane fusion are inhibited by the replacement of its membrane-spanning domain. J Biol Chem 285, 14681-14688.

Li, L., Vorobyov, I., MacKerell, A.D. Jr, and Allen, T.W. (2008). Is arginine charged in a membrane? Biophys J 94, L11-L13.

Liu, S., Kondo, N., Long, Y., Xiao, D., Iwamoto, A., and Matsuda, Z. (2010). Membrane topology analysis of HIV-1 envelope glycoprotein gp41. Retrovirology 7, 100.

Melikyan, G.B. (2008). Common principles and intermediates of viral protein-mediated fusion: the HIV-1 paradigm. Retrovirology 5, 111.

Miyauchi, K., Curran, A.R., Long, Y., Kondo, N., Iwamoto, A., Engelman, D.M., and Matsuda, Z. (2010). The membranespanning domain of gp41 plays a critical role in intracellular trafficking of the HIV envelope protein. Retrovirology 7, 95.

Miyauchi, K., Komano, J., Yokomaku, Y., Sugiura, W., Yamamoto, N., and Matsuda, Z. (2005). Role of the specific amino acid sequence of the membrane-spanning domain of human immunodeficiency virus type 1 in membrane fusion. J Virol 79, 4720-4729.

Morton, H.C., van den Herik-Oudijk, I.E., Vossebeld, P., Snijders, A., Verhoeven, A.J., Capel, P.J., and van de Winkel, J.G. (1995). Functional association between the human myeloid immunoglobulin A Fc receptor (CD89) and FcR gamma chain. Molecular basis for CD89/FcR gamma chain association. J Biol Chem 270, 29781-29787.

Owens, R.J., Burke, C., and Rose, J.K. (1994). Mutations in the membrane-spanning domain of the human immunodeficiency virus envelope glycoprotein that affect fusion activity. J Virol 68 , 570-574.

Pietschmann, T., Zentgraf, H., Rethwilm, A., and Lindemann, D. (2000). An evolutionarily conserved positively charged amino acid in the putative membrane-spanning domain of the foamy virus envelope protein controls fusion activity. J Virol 74, 4474-4482.

Reeves, J.D., Gallo, S.A., Ahmad, N., Miamidian, J.L., Harvey, P.E., Sharron, M., Pohlmann, S., Sfakianos, J.N., Derdeyn, C.A., Blumenthal, R., et al. (2002). Sensitivity of HIV-1 to entry inhibitors correlates with envelope/coreceptor affinity, receptor density, and fusion kinetics. Proc Natl Acad Sci U S A 99, 16249-16254.

Rowell, J.F., Stanhope, P.E., and Siliciano, R.F. (1995). Endocytosis of endogenously synthesized HIV-1 envelope protein. Mechanism and role in processing for association with class II MHC. J Immunol 155, 473-488.

Shang, L., and Hunter, E. (2010). Residues in the membranespanning domain core modulate conformation and fusogenicity of the HIV-1 envelope glycoprotein. Virology 404, 158-167.

Shang, L., Yue, L., and Hunter, E. (2008). Role of the membranespanning domain of human immunodeficiency virus type 1 envelope glycoprotein in cell-cell fusion and virus infection. $J$ 
Virol 82, 5417-5428.

Weiss, C.D. (2003). HIV-1 gp41: mediator of fusion and target for inhibition. AIDS Rev 5, 214-221.

Welman, M., Lemay, G., and Cohen, E.A. (2007). Role of envelope processing and gp41 membrane spanning domain in the formation of human immunodeficiency virus type 1 (HIV-1) fusion-competent envelope glycoprotein complex. Virus Res 124, 103-112.

Wilk, T., Pfeiffer, T., Bukovsky, A., Moldenhauer, G., and Bosch, V. (1996). Glycoprotein incorporation and HIV-1 infectivity despite exchange of the gp160 membrane-spanning domain. Virology 218,
269-274.

Wyss, S., Dimitrov, A.S., Baribaud, F., Edwards, T.G., Blumenthal, R., and Hoxie, J.A. (2005). Regulation of human immunodeficiency virus type 1 envelope glycoprotein fusion by a membraneinteractive domain in the gp41 cytoplasmic tail. J Virol 79, $12231-12241$.

Yang, C., Spies, C.P., and Compans, R.W. (1995). The human and simian immunodeficiency virus envelope glycoprotein transmembrane subunits are palmitoylated. Proc Natl Acad Sci U S A 92, 9871-9875. 